OPEN ACCESS

Edited by:

Kenneth Curley,

latrikos Research and Development Solutions, LLC, United States

Reviewed by:

Tom M. McMillan, University of Glasgow,

United Kingdom

Edwin Arthur Shores,

Macquarie University, Australia

*Correspondence:

Erin S. Kenzie

erin.kenzie@pdx.edu

Specialty section: This article was submitted

to Neurotrauma,

a section of the journal

Frontiers in Neurology

Received: 21 November 2017 Accepted: 14 March 2018

Published: 04 April 2018

Citation:

Kenzie ES, Parks EL, Bigler ED, Wright DW, Lim MM, Chesnutt JC,

Hawryluk GWJ, Gordon W and

Wakeland W (2018) The Dynamics

of Concussion: Mapping

Pathophysiology, Persistence, and

Recovery With Causal-Loop

Diagramming.

Front. Neurol. 9:203.

doi: 10.3389/fneur.2018.00203

\section{The Dynamics of Concussion: Mapping Pathophysiology, Persistence, and Recovery With Causal-Loop Diagramming}

Erin S. Kenzie ${ }^{1 *}$, Elle L. Parks ${ }^{1}$, Erin D. Bigler ${ }^{2}$, David W. Wright ${ }^{3}$, Miranda M. Lim ${ }^{4,5}$, James C. Chesnutt ${ }^{6}$, Gregory W. J. Hawryluk ${ }^{7}$, Wayne Gordon ${ }^{8}$ and Wayne Wakeland ${ }^{1}$

${ }^{1}$ Systems Science Program, Portland State University, Portland, OR, United States, ${ }^{2}$ Department of Psychology and Neuroscience Center, Brigham Young University, Provo, UT, United States, ${ }^{3}$ Department of Emergency Medicine, Emory University School of Medicine, Atlanta, GA, United States, ${ }^{4}$ Sleep Disorders Clinic, Division of Hospital and Specialty Medicine, Research Service, VA Portland Health Care System, Portland, OR. United States, ${ }^{5}$ Departments of Neurology, Medicine, and Behavioral Neuroscience, Oregon Institute of Occupational Health Sciences, Oregon Health \& Science University, Portland, OR, United States, ${ }^{6}$ TBI/Concussion Program, Orthopedics \& Rehabilitation, Neurology and Family Medicine, Oregon Health \& Science University, Portland, OR, United States, ${ }^{7}$ Department of Neurosurgery, University of Utah, Salt Lake City, UT, United States, ${ }^{8}$ Department of Rehabilitation Medicine, Icahn School of Medicine at Mount Sinai, New York, NY, United States

Despite increasing public awareness and a growing body of literature on the subject of concussion, or mild traumatic brain injury, an urgent need still exists for reliable diagnostic measures, clinical care guidelines, and effective treatments for the condition. Complexity and heterogeneity complicate research efforts and indicate the need for innovative approaches to synthesize current knowledge in order to improve clinical outcomes. Methods from the interdisciplinary field of systems science, including models of complex systems, have been increasingly applied to biomedical applications and show promise for generating insight for traumatic brain injury. The current study uses causal-loop diagramming to visualize relationships between factors influencing the pathophysiology and recovery trajectories of concussive injury, including persistence of symptoms and deficits. The primary output is a series of preliminary systems maps detailing feedback loops, intrinsic dynamics, exogenous drivers, and hubs across several scales, from micro-level cellular processes to social influences. Key system features, such as the role of specific restorative feedback processes and cross-scale connections, are examined and discussed in the context of recovery trajectories. This systems approach integrates research findings across disciplines and allows components to be considered in relation to larger system influences, which enables the identification of research gaps, supports classification efforts, and provides a framework for interdisciplinary collaboration and communication-all strides that would benefit diagnosis, prognosis, and treatment in the clinic.

Keywords: concussion, traumatic brain injury, systems science, complexity, recovery, causal-loop diagram, models of injury, systems medicine 


\section{INTRODUCTION}

Concussion, also known as mild traumatic brain injury (mTBI), ${ }_{1}^{1}$ is a significant public health issue responsible for a variety of cognitive, emotional, and somatic symptoms and deficits (3). It is unclear why some individuals appear to recover relatively quickly while others suffer prolonged symptoms and impairments (4-7). Robust clinical means of diagnosis, prognosis, and treatment are also lacking (8-11). Research is hindered by an inadequate classification system for traumatic brain injury (TBI) (12), "poor" study quality $(13,14)$, disagreement about appropriate inclusion and exclusion criteria for concussion $(8,15)$, and an incomplete understanding of underlying pathophysiology $(16-18)$. The heterogeneity and complexity seen in concussion further complicate research, particularly efforts to individualize treatment (19-22).

The study and clinical care of concussion spans many disciplines. Integrating research findings into a common framework would yield benefits for interdisciplinary communication, but such integration faces significant challenges. Diverse specialties use different definitions of concussion, models of injury, and measures of progress toward recovery (23). Translation of findings from animal models is particularly challenging when studying subtle changes in human consciousness (20). Initial efforts to improve data agreement, such as the Common Data Elements ${ }^{2}$ and Federal Interagency Traumatic Brain Injury Research, ${ }^{3}$ have made important strides toward interdisciplinarity, but are constrained by the lack of a shared explanatory model upon which to base a new system of classification (24). Recent biopsychosocial models combining multiple variables of concussion recovery have been proposed (25-27), but their linear formulation and limited scope fall short of capturing the complex, interdependent network of factors and nonlinear recovery trajectories often seen with concussion. A recent article published by researchers associated with the International Initiative for Traumatic Brain Injury Research (22) highlights the importance of "multidimensional approaches" and prognostic models for improving clinical outcomes.

Systems science is an interdisciplinary field of study offering diverse methods and theories for the study of complex systems. A key idea underlying the systems approach is an emphasis on the interconnections between system components and how mutual causality impacts overall system behavior or performance. These methods can be used to better understand complex public health issues by providing ways of visualizing and analyzing systems of interest $(28,29)$. By synthesizing information from diverse stakeholders and fields, they can serve as an organizing framework for current knowledge and support the development of a shared understanding of complex phenomena (30). In recent years, this approach has been increasingly applied to biomedical and public

${ }^{1}$ Here, we choose to use these terms interchangeably, but recognize the ongoing debate in the literature $(1,2)$. Although concussion is arguably more colloquial than $m T B I$, it is flexible enough to accommodate the development of a new classification system (whereas mTBI is tied to the Glasgow Coma Scale). We do intend to be inclusive of complicated mild cases with prolonged recoveries.

${ }^{2} \mathrm{https} / /$ www.commondataelements.ninds.nih.gov/ (Accessed: March 17, 2018).

${ }^{3}$ https://fitbir.nih.gov/ (Accessed: March 17, 2018). health issues such as obesity (31), drug diversion and abuse (32), and depression (33).

Previous research conducted by several of the current authors examined the state of knowledge about concussion through a systems lens and identified key variables relevant to recovery across multiple scales (19). The current research builds upon that work by producing a detailed systems model of concussion pathophysiology and persistence or recovery of symptoms, with a focus on the feedback relationships underlying nonlinear system behavior. This paper introduces key systems concepts to the TBI community and provides an opportunity to examine whether useful insight can be generated using a novel systems mapping approach.

Treating concussion injury and recovery as a complex, interdependent system of physiological, experiential, and social variables influenced by a heterogeneous array of personal and injury characteristics is a significant shift from conventional approaches to medical research in which variability is minimized in order to identify correlations between a small number of variables. Working in conjunction with traditional reductionist research that can identify individual relationships from controlled experiments and newer big-data efforts that can identify patterns in large sets of data, systems science methods enable a big-picture perspective that can inform research. Because clinical and research disciplines in medicine are highly specialized, opportunities to develop holistic perspectives are rare. Systems science methods are particularly well suited to a key challenge in brain injury research: understanding mechanisms underlying heterogeneous recovery trajectories, in order to improve clinical prediction models and classification of patients at various time points in recovery. By analyzing how variables interrelate to enable symptom resolution or persistence, the complex nature of concussion can be better understood.

\section{MATERIALS AND METHODS}

To address current questions about heterogeneity, classification, and lack of a shared explanatory framework in concussion, the Brain Trauma Foundation convened an interdisciplinary panel of researchers and clinicians in the field of TBI as part of the Brain Trauma Evidence-based Consortium (B-TEC). Model development was led by a core methods team with expertise in systems science and neuroscience. The model was developed iteratively, with a high degree of involvement from TBI researchers and clinicians. Several researchers provided close support throughout the multi-year process and are included as coauthors on the current paper, while a series of in-person meetings allowed input from B-TEC investigators. Presentation of in-progress models at several conferences allowed for feedback from broader communities of TBI researchers and systems modelers. Rounds of literature review and individual interviews with 26 experts (Table S1 in Supplementary Material) further guided model development. Interviews were semi-structured, conducted by members of the core methods team, and typically lasted 60-90 min. Transcriptions of the interviews were coded and analyzed for relevant content. Model revisions were made in an iterative fashion as new information was gathered. 
Review of the literature was informed by guidance from experts and needs that were identified during the process of model building. The review of published, peer-reviewed literature was extensive, though not systematic; due to known data quality issues with the existing literature (34), the choice was made to take an inclusive approach. To build a comprehensive model using only studies meeting the highest methodological standards would have been impossible since very few such studies exist (34). Furthermore, including a wide variety of types of peer-reviewed research allows for a richer, albeit more speculative, view of the system to emerge.

In an earlier phase of the project, the team outlined the application of a systems approach to concussion and developed systems diagrams outlining key variables across four emergent, interconnected scales: cellular, network, experiential, and social (19). For the current phase, the team further expanded and specified these models as causal-loop diagrams (CLDs) to describe interconnections between system variables in more detail. CLDs use a simple notation to map hypothesized causal relationships between variables in aggregate quantities (35). This method is particularly useful for revealing feedback loops, which often generate nonlinear dynamics in complex systems.

Unlike statistical models extrapolated or imputed from correlations in relevant data, systems models are often built in a top-down fashion based on causal hypotheses of how the systems are thought to operate. Such a model serves as a reflection of the knowledge and assumptions held by a person or group-a shared mental model in the systems literature (36). When empirical or theoretical knowledge about the target system is incomplete, a systems model can identify where to focus attention for future empirical investigation.

The goal of building this model is to depict relationships between key variables influencing concussion pathophysiology and symptomatology across multiple scales, with a focus on identifying endogenous feedback mechanisms that shape recovery trajectories. While the heterogeneity of concussion implies that one static model cannot be universally applicable across all patients, an effort was made to include variables and relationships common to many cases. The team identified primary system components from interview data and review of the literature, then, modeled them at a scale determined by their connections to other system components. This resulted in a model describing pathophysiology and recovery at several scales. Priority was given to articulating how and why post-concussive symptoms might persist over time.

Due to evidence indicating distinct pathophysiological mechanisms underlying blast injury (16, 37-39), the decision was made to focus exclusively on concussion (or mTBI) caused by blunt impact or accelerative/decelerative forces. Also excluded were penetrating injuries, as they are generally associated with more severe TBI, including more focal deficits related to the specific location of injury.

Documentation of supporting evidence was done alongside model building, and was ultimately compiled in the Evidence Table (Table S2 in Supplementary Material).

After building the model, the core team conducted loop analysis to identify feedback loops and key connections. Supplementary diagrams were created to communicate noteworthy patterns in loop structure.

\section{RESULTS: CLD}

The CLD shown in Figure 1 provides an interdisciplinary, multiscale depiction of key feedback dynamics related to concussion, including persistent post-concussive syndrome. The model includes injury to the brain from blunt impact or accelerative/ decelerative forces, but is not intended to describe blast injury. The diagram shows a system that is highly complex, with many feedback loops and connections between subsystems operating at different biological and time scales. The model is intended as a preliminary demonstration of this method and only captures a portion of the immense complexity of concussion recovery. An interactive, web-based reproduction of Figure 1 was generated using Kumu (40) and can be found at www.dynamicsofconcussion.com.

The complex, highly interconnected nature of the system of factors relevant to concussion pathophysiology and recovery is readily apparent in Figure 1. The large number of incoming and outgoing arrows connected to many of the variables indicates a system in which causality is shared across a diverse set of factors. After providing an orienting narrative of the systems model, specific aspects such as feedback dynamics, drivers, hubs, and boundaries will be presented.

\section{Model Narrative}

From left to right, Figure 1 shows variables at increasing time scales and at several scales of biological organization: cellular, network, experiential, and social. This section describes in narrative form the relationships seen in Figure 1. Note that neither the model nor the corresponding narrative is exhaustive. Rather, they reference only system components identified during the course of our research.

\section{Cellular}

Immediately following impact to the brain (from either a blunt force or rapid acceleration/deceleration of the head), biomechanical stretch/strain effects from deformation may alter the cytoskeletal structure of neurons, glia, vasculature, and the structural extracellular matrix (41). This disruption initiates a variety of acute neuroanatomic, neurotransmitter, neurometabolic, inflammatory, and vascular processes at the cellular level (42). Physical alteration from transient disruption of brainstem-cortical connections may include loss of consciousness if rotational forces transverse to the brainstem are present $(43,44)$. Excessive glutamate and ionic flux contribute to an energy crisis, which can result in a prolonged hypometabolic state following injury (42). Calcium dysregulation persists longer than other ionic disruptions and can cause mitochondrial dysfunction and oxidative stress, and exacerbate the cellular energy imbalance (42). The byproducts of various cellular processes can create a pathological microenvironment, which puts further stress on cellular networks (45). Physical damage to astrocyte and microglial cells, along with their activation in response to injury, can cause permeability in the blood-brain barrier whereby peripheral leukocytes and other 


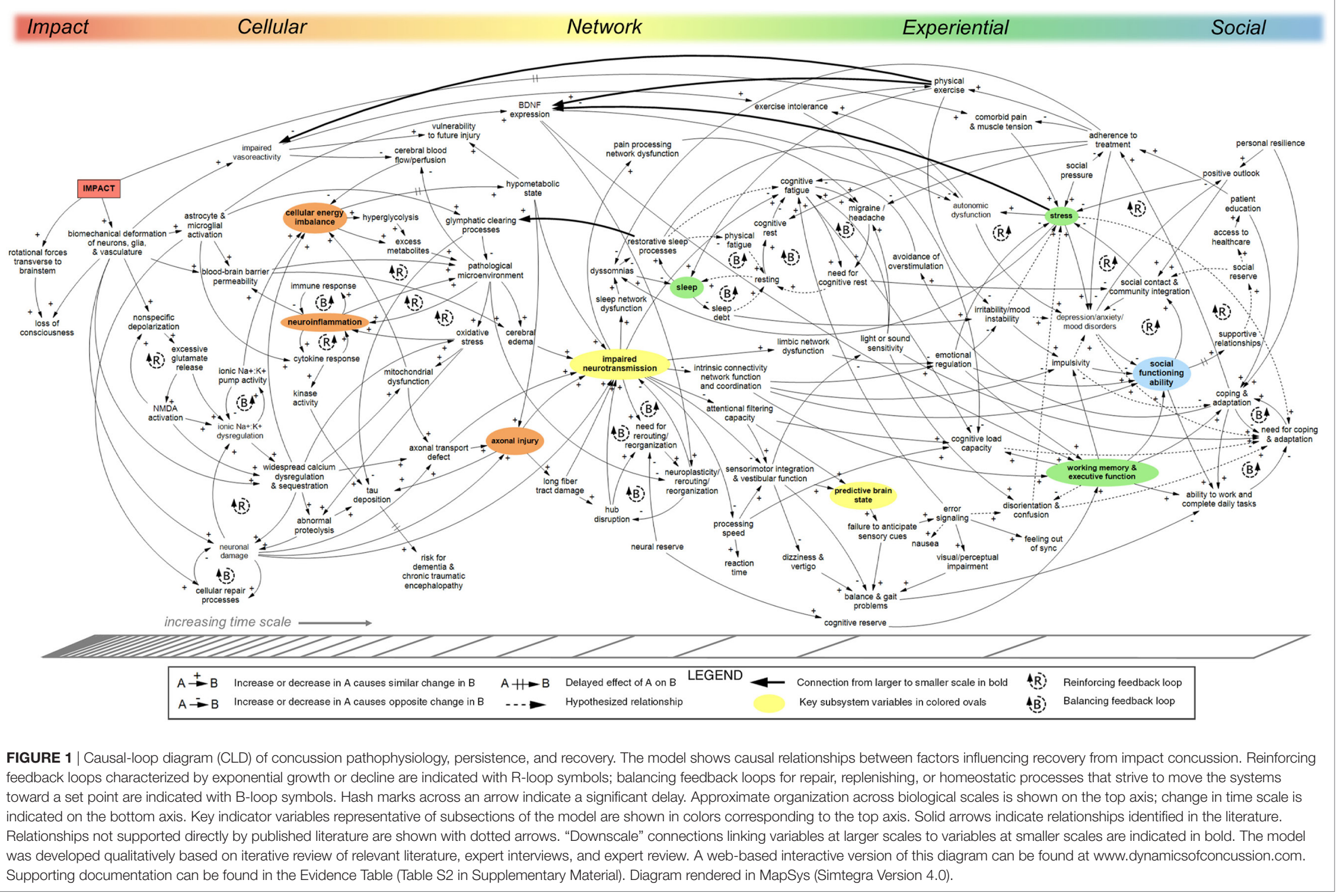


compounds contribute to a pathological microenvironment causing neuroinflammation $(46,47)$. If neurometabolism is disrupted for an extended period of time, mechanisms of neuronal damage are initiated due to calcium sequestration and abnormal proteolysis (42), as well as axonal injury, via extended hypometabolism and various defects in axonal transport (48-52).

\section{Network}

Disruption at the cellular level directly impairs neurotransmission, which causes both generalized as well as focal functional impairment in any number of neural networks, depending on the location of impairment, the cell types impacted, and the largescale population dynamics of local and global neuronal ensembles (53-55). Impaired neurotransmission serves as a key hub in our model and a point of emergence between cellular-scale processes and neural networks $(56,57)$. Using impaired neurotransmission as an aggregate variable excludes the spatially localized information gleaned from neuroimaging and histological studies. While these approaches are crucial to better understanding injury heterogeneity, the current project focuses instead on the causes and effects of neurotransmission impairment in order to illustrate system-level nonlinear feedback dynamics responsible for symptom persistence and recovery across heterogeneous concussive injuries.

Impaired neurotransmission is exacerbated by damage to long fiber tracts and hub disruption, and is ameliorated by processes of neuroplasticity, rerouting, and reorganization (58). The manner in which network dysfunction is repaired or manifests into symptom sequelae evolves throughout recovery; for example, distinct patterns of cerebral blood flow and white matter microstructure have been shown to relate to different symptoms at different time points post-injury, further illustrating the dynamic and ever-shifting relationships between network and experiential scales of concussion recovery $(5,59-61)$.

Seamless interaction between networks in the connectome is necessary for integrated conscious experience. For example, the salience network provides the critical function of switching activation between the default-mode network and the central executive network $(62,63)$. This allows the individual to adjust between states of quiescence and rest and goal-directed tasks (see Figure 2).

Post-concussive symptoms often reflect network dysfunction not only within the primary network related to a given symptom expression but also via the primary network's temporal or structural coordination with all other networks (65). Network correlates of single symptoms may shift and evolve throughout recovery, as will detection by the individual experiencing the symptom after sustaining a concussion.

\section{Experiential}

Network dysfunctions in concussion manifest in the individual as a variety of somatic, cognitive, and affective symptoms, such as mood disruptions, sleep disturbances, migraine/headache, impaired sensorimotor integration, and reduced cognitive processing speed $(59,64,66-70)$. Abnormalities and asynchrony in signal processing result in cognitive fatigue, problems with sensorimotor integration, error signaling, and feeling out of

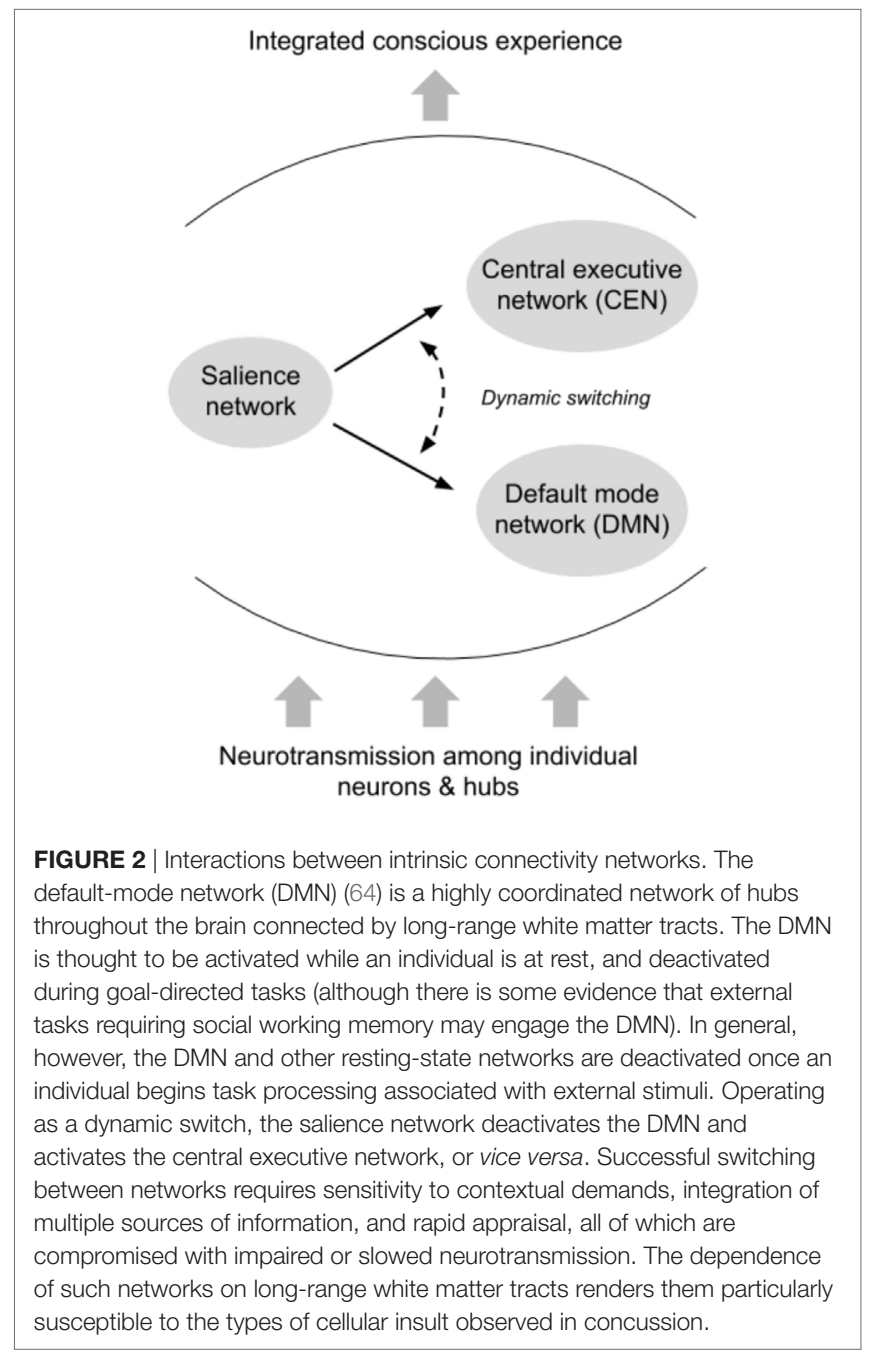

sync (71). Sleep dysfunction as a post-concussion symptom is commonplace, which has a direct bearing on all brain networks because sleep is critical for glymphatic clearing of brain waste and provides a key feedback to the cellular scale by ameliorating local pathological microenvironments (72-74). Dysfunction in sleep patterns may lead to reduced cognitive load capacity and increased mood instability, and could interfere with social engagement-all problems experienced by patients with residual post-concussion symptoms (75).

Mood changes can originate in disruption of limbic and frontal networks and have social and behavioral effects (76-79). Psychological stress has wide-ranging consequences, from impaired brain-derived neutrophic factor (BDNF) expression and autonomic dysfunction to problems with sleep, mood, cognition, and coping $(45,80)$. Working memory and executive function have additional social and behavioral effects (81). Cognitive load capacity is affected by a variety of factors in the model, such as stress, exercise intolerance, need for cognitive rest, sleep, processing speed, and working memory, and executive function (82). Light or sound sensitivity can further exacerbate migraine headache, cognitive fatigue, and disorientation/confusion $(83,84)$. 


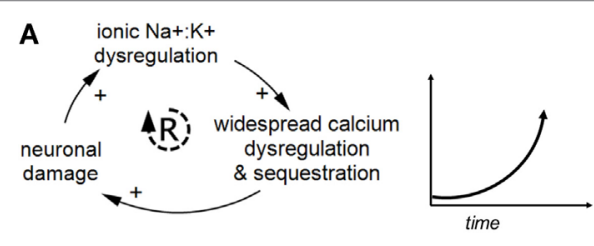

B ionic $\mathrm{Na}+\mathrm{K}+$

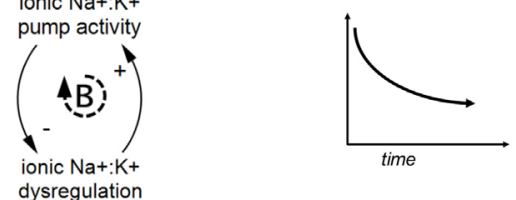

FIGURE 3 | Examples of reinforcing and balancing feedback. Panel (A) shows a reinforcing feedback loop and a corresponding graph of exponentially increasing behavior over time. Panel (B) shows a balancing feedback loop and a corresponding graph of decreasing behavior over time toward an internal set point, based on ionic pump activity attenuating ionic dysregulation. Reinforcing and balancing feedback are the two types of feedback loops found in complex systems.

Problems with sensorimotor integration and vestibular function can cause issues with balance and gait, dizziness, and disruption in predictive timing of sensory input $(82,85)$. Disruption in the predictive brain state causes error signaling and its downstream effects (71).

\section{Social}

Cognitive and mood dysfunctions can impair an individual's ability to function socially, which over time can erode the support they receive from strong personal relationships and integration in their communities (81). Strong social support and personal resilience are included in the model as positively impacting treatment, and coping and adaptation $(86,87)$. Social pressure, however, increases stress and interferes with adherence to treatment in the model. The model also includes several neuroprotective processes, such as cognitive rest, physical exercise, avoidance of overstimulation, and other treatment for headache, pain, and sleep dysfunction.

\section{Feedback}

A key benefit to causal-loop diagramming is that it makes feedback relationships explicit. Feedback loops take two forms: reinforcing and balancing (see Figure 3). Reinforcing loops are "vicious" or "virtuous" cycles or cascades of exponential growth or decline. These loops can push the system out of balance in one direction or another when left unchecked. In contrast, balancing loops indicate repair, replenishing, homeostatic, or otherwise restorative processes and can be viewed as influencing progress toward a set point, goal, or neutral state.

Feedback loops exist both within specific biological scales and across multiple scales in the model. Figure 4 provides a generic illustration of feedback within and across scales in concussion. Upward arrows from smaller to larger scales can indicate emergence, as with individual neurons assembling into networks [see Ref. (19) for further discussion].

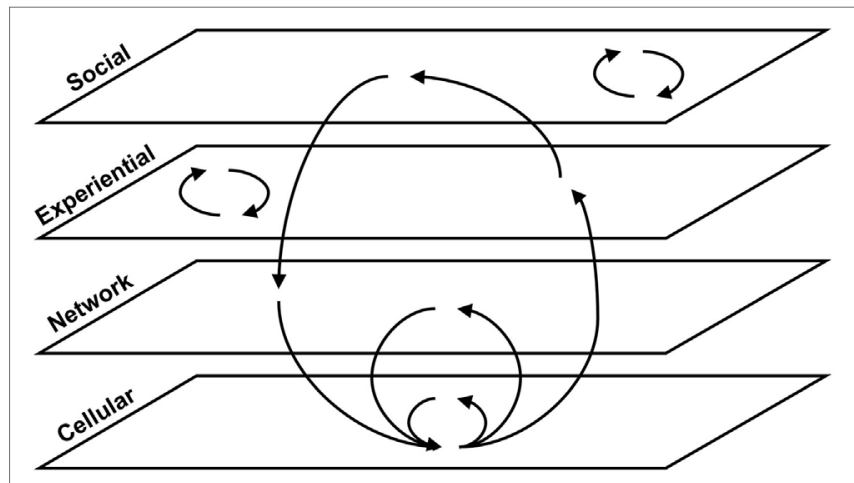

FIGURE 4 | Within-scale and cross-scale feedback loops. Generic feedback loops are shown across four scales of organization relevant to concussion pathophysiology and recovery. Some loops occur within a given scale, and others span multiple scales.

\section{Within-Scale Loops}

Short loops existing within one scale are denoted with $\mathrm{R}$ or B-loop icons in Figure 1. These loops indicate processes that often fall within the purview of a particular subfield of medicine or research discipline. At the cellular level, balancing loops for ionic pump activity, cellular repair, and immune response help to address damage and imbalance in the cellular milieu. Reinforcing loops related to glutamate release and ionic dysregulation show the cascading processes behind the neurometabolic cascade identified in concussion. A series of reinforcing loops around neuroinflammation show how inflammation can trigger processes that further exacerbate inflammation. A loop between the blood-brain barrier, pathological microenvironment, and neuroinflammation plays a particularly important role in prolonged recovery $(46,47,88,89)$.

Larger within-scale loops not marked with loop icons can also be found in Figure 1. Figure 5 shows a series of nested reinforcing feedback loops within the cellular scale describing relationships between the metabolic, ionic, and neuronal subsystems. Individual feedback loops are indicated in this diagram to demonstrate the large number of loops that emerge from a relatively small number of variables and connections.

Within the network level, several short balancing loops describe how neuroplasticity, neuronal rerouting, and reorganization work to rectify impaired neurotransmission, particularly hub disruption. In neural networks, if the original primary pathways cannot come back online, then backup systems attempt to take over, depending on the redundancy and parallel connections within any given network $(58,90)$. Successful reorganization is determined in part by neural reserve (91).

Sleep and cognitive fatigue feature a cluster of tightly connected balancing loops at the center of Figure 1. Figure 6 shows the interconnections between the sleep/fatigue, mood, stress, and autonomic subsystems. At the right side of Figure 1, these subsystems interlink with social variables, illustrating how emotional and social problems can compound one another. Figure 6 only depicts the connections described in Figure 1 and is not intended to comprehensively describe sleep dynamics. 


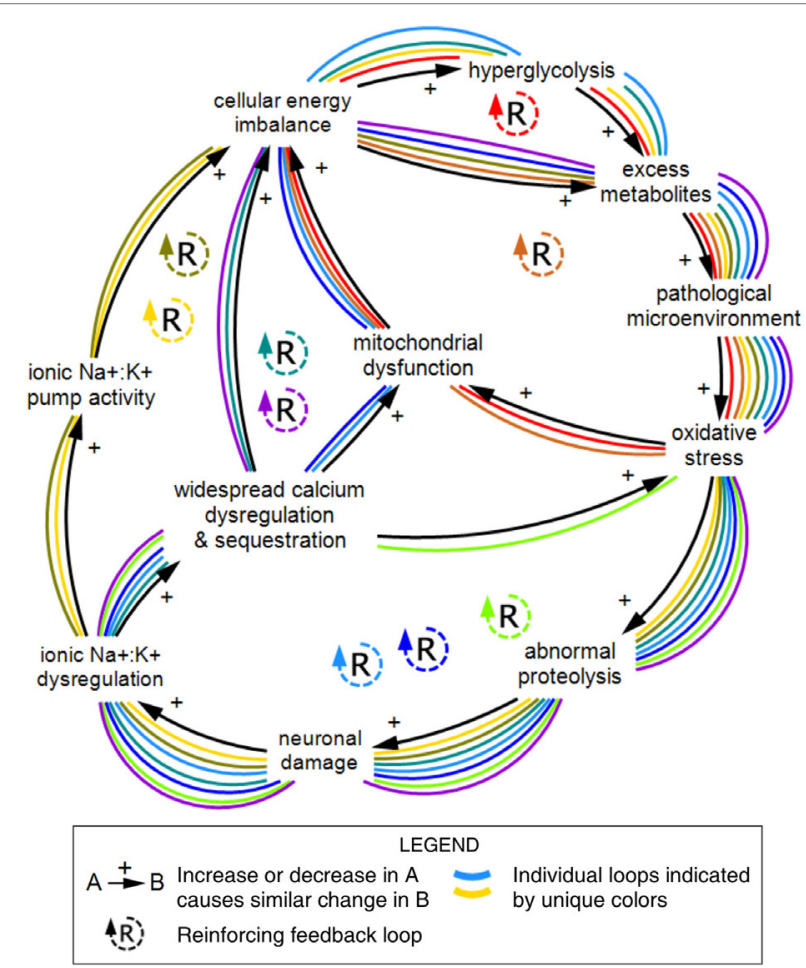

FIGURE 5 | Nine feedback loops within the cellular scale of concussion. A series of reinforcing loops across the metabolic, ionic, and neuronal subsystems demonstrate the large number of feedback relationships that emerge from connections between a relatively small number of variables. The reinforcing structure of these relationships indicates compounding effects over time. Individual feedback loops are marked with unique colors. Diagram rendered in MapSys.
A substantial number of loops within the experiential scale pertain to mechanisms of coping and adaptation that constitute balancing processes in the system. A series of multi-component balancing loops involving coping and adaptation on the righthand side of Figure 1 are highlighted in Figure 7.

In several places in the model, a variable associated with a restorative process takes effect due to an increased need for repair or restoration, creating a balancing loop. For example, a need for cognitive rest results in more resting, which in turn decreases the need for rest. Distinguishing the need for something from the variable itself illustrates an endogenous goal-seeking process and makes distinct relationships with other subsystems explicit. Note that not all such processes have been specified in Figure 1.

\section{Cross-Scale Loops}

Including variables existing at multiple scales in one comprehensive model enables the identification of cross-scale feedback loops. Larger cross-scale feedback loops can be difficult to recognize, but can be critical to the structure of the system and, therefore, to how it operates. Many of the arrows in the concussion model (Figure 1) go from left to right, indicating emergence from smaller to larger scales (19). Several key arrows instead go from right to left, linking variables at the larger scale with variables at the smaller scale (indicated in bold in Figure 1). These "downscale" connections enable experiential and social processes to affect cellular and molecular processes, thereby creating a significant number of feedback loops in the model (summarized in Figure 8).

The connection between restorative sleep processes and glymphatic clearing in the concussion model (Figure 1) is particularly

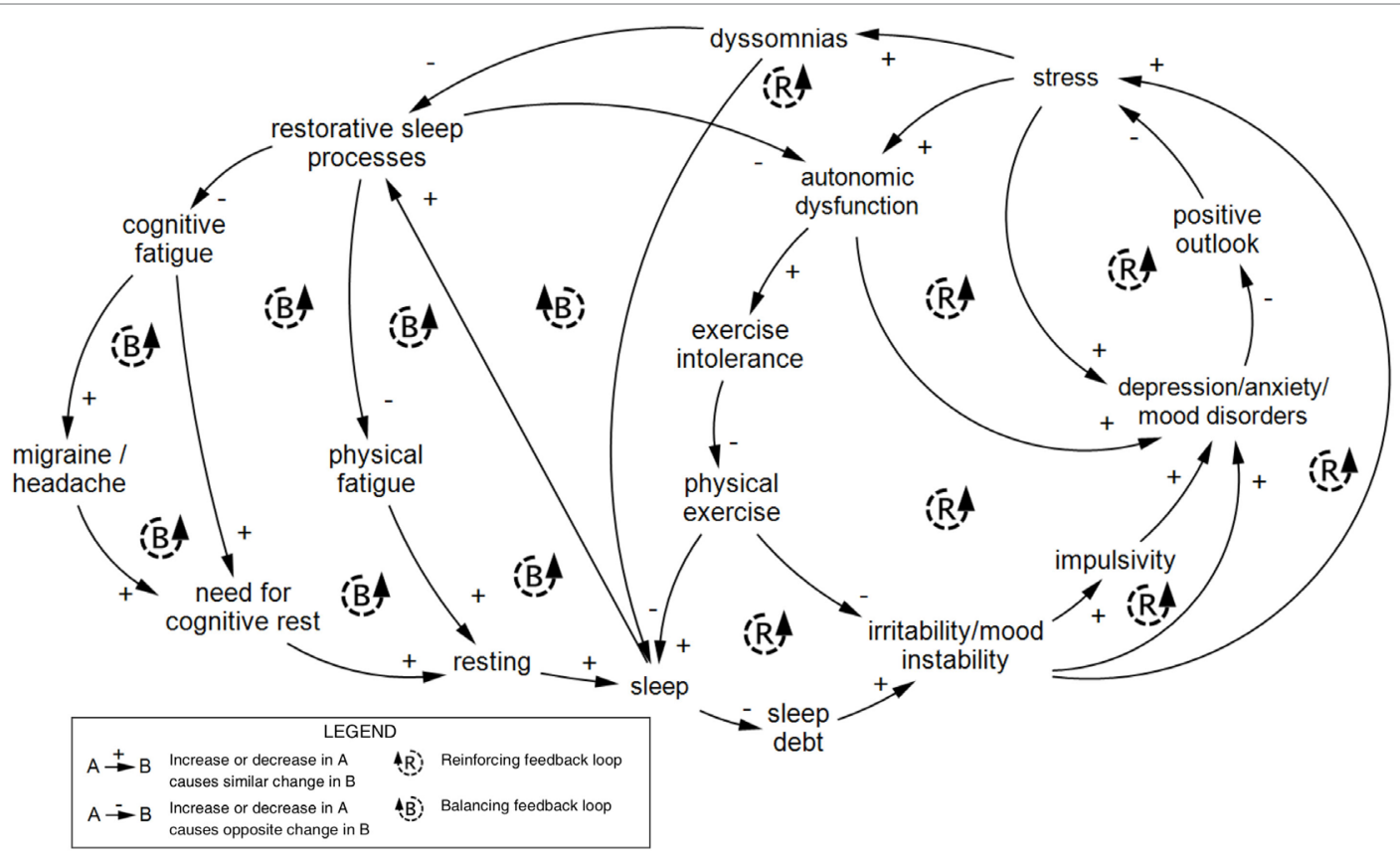

FIGURE 6 | Feedback loops within the experiential scale of concussion. A series of nested feedback loops across the sleep/fatigue, autonomic, mood, and stress subsystems within the experiential scale are shown to illustrate the interconnectedness of variables across subsystems. This series of loops was reproduced from Figure 1. Diagram rendered in MapSys. 


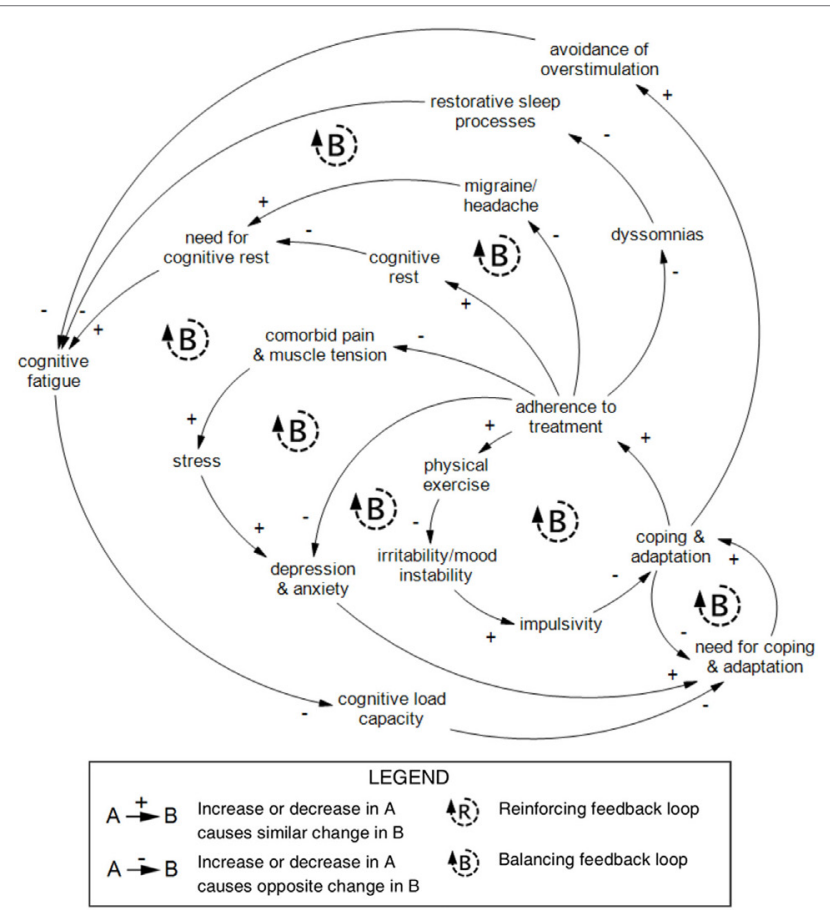

FIGURE 7 | Balancing loops related to coping and adaptation within the experiential scale in recovery from concussion. This series of nested balancing feedback loops was reproduced from Figure 1. A core loop distinguishes coping and adaptation from the need for coping and adaptation. Coping and adaptation lead to two behaviors: avoidance of overstimulation and adherence to treatment. Avoidance of overstimulation reduces cognitive fatigue. Access and quality of medical treatment and advice will vary between cases, but targeted treatments may be prescribed by a clinician to increase cognitive rest or reduce migraine/headache, cognitive fatigue, comorbid pain and muscle tension, stress, depression and anxiety, irritability/mood instability, and impulsivity. Diagram rendered in Mapsys.

critical because it enables a series of balancing feedback loops pertaining to symptoms, network disruption, and impaired neurotransmission, the primary hub. Such loops feature a variety of neural networks and symptoms, but all tend to follow a similar basic structure (summarized in loop B1 in Figure 8A). Because sleep is a universal process, this loop provides the primary crossscale restorative mechanism in the model.

Physical exercise also introduces balancing loops via its effect on expression of BDNF and impaired vasoreactivity (Figure 8B). An additional connection from a higher to lower scale is the link between stress and BDNF expression, which introduces several reinforcing loops in which stress exacerbates symptoms and slows recovery (Figure 8C). Problems with social functioning can prompt coping and adaptation, but also worsen stress and its effects (Figure 8D).

The downscale connections included in this model (pertaining to sleep, physical exercise, and stress) describe behaviors that have known biophysical components. As the psychological, emotional, and social dimensions of recovery are increasingly recognized $(19,81,92)$, additional feedback relationships will be identified.

\section{Loop Dominance}

When loops interconnect, as shown in Figure 8, their relative influence can change over time, a phenomenon referred to as shifting loop dominance (35). For example, if the balancing loops in Figure $\mathbf{8}$ are dominant, impaired neurotransmission-and therefore symptoms-will be on a decreasing trajectory as the various coping and adaptation strategies take effect. But if the individual experiences a significant increase in stress due to either an increase in concussion symptoms or an unrelated stressor, for example, the reinforcing loops could become dominant, which would disrupt the patient's recovery. Effective treatment could offset the effects of the stressful event and permit the balancing loops to once again dominate so that symptoms resume their downward trajectory. Figure 9 shows a sample recovery trajectory influenced by shifting loop dominance.

Examining loop structure and dominance within systems can facilitate greater understanding of nonlinear behavior of key variables of interest over time. It can also help explain how the system can get "stuck" in certain patterns, which in the case of concussion reflect the persistence of symptoms and deficits. For concussion recovery, which can be prolonged, unpredictable, and highly dependent on individual characteristics and context, this type of analysis is particularly suitable.

\section{Drivers}

System drivers in a CLD are exogenous elements that affect variables in the system but are not themselves affected; they can be thought of as operating at the system boundary. Due to the large number of relationships already shown in the concussion CLD, only a few of the potential drivers are shown: impact, neural reserve, social pressure, and personal resilience. These elements represent aspects of the individual's context and personal history that remain largely unchanged by variables endogenous to the system.

\section{Personal and Injury Characteristics}

Concussion is highly heterogeneous, and myriad personal and injury characteristics shape recovery trajectories $(21,25)$. Several such characteristics were included in the concussion model (Figure 1) as drivers, as listed above. Although a comprehensive inventory of individual characteristics is outside the scope of this paper, presentation of the concussion model allows for the identification of several ways in which these characteristics interact with the system.

Some individual characteristics affect baseline levels of specific variables; for example, recent prior concussions could cause a degree of pre-injury hypometabolism in some individuals (42), while a history of migraines, depression, or sleep disruption would similarly alter baselines for those variables. Individuals with altered baseline levels could be more sensitive to certain feedback loops within the CLD, and as a result, be more or less prone to magnified symptoms following TBI. Also note that the non-specificity of symptoms of concussion complicates attribution in the absence of pre-injury baseline data.

Other characteristics could affect the efficiency or time course of certain processes, such as the immune response or glymphatic clearing, or affect the threshold at which symptoms occur. Certain 


\section{A Sleep helps repair neurotransmission}

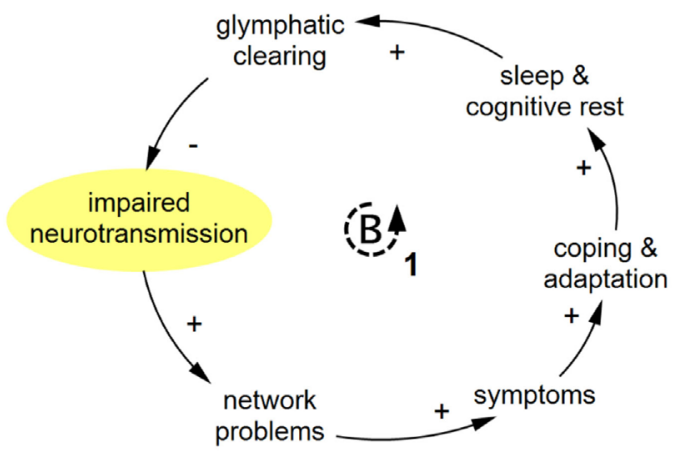

C But stress exacerbates symptoms

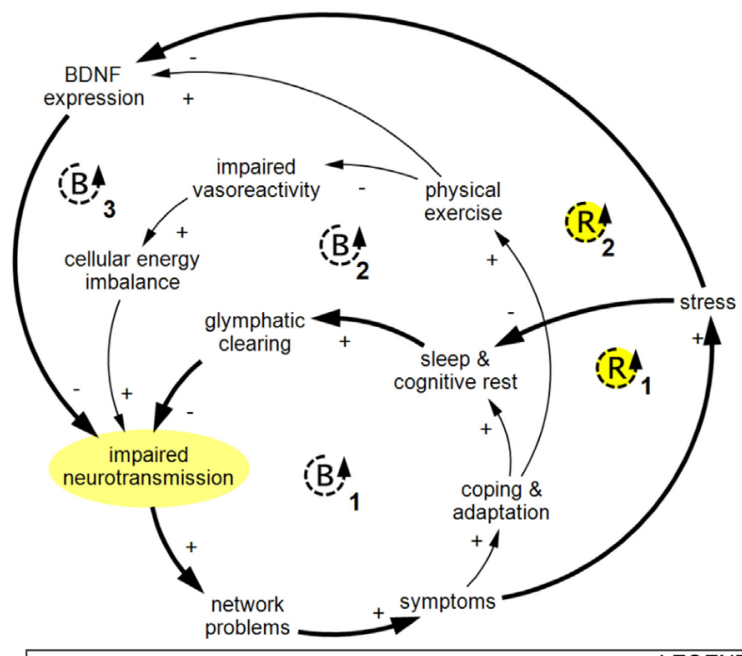

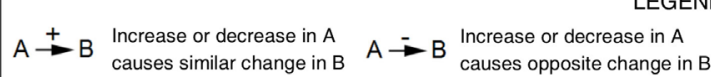

B Physical exercise can also be restorative

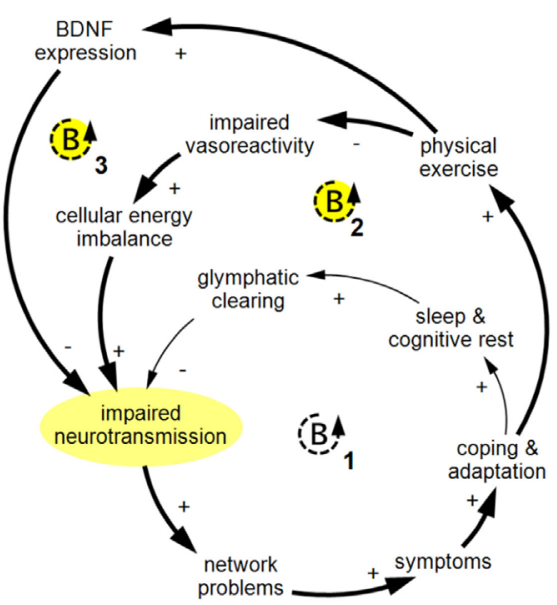

D Social functioning problems necessitate coping but exacerbate stress effects

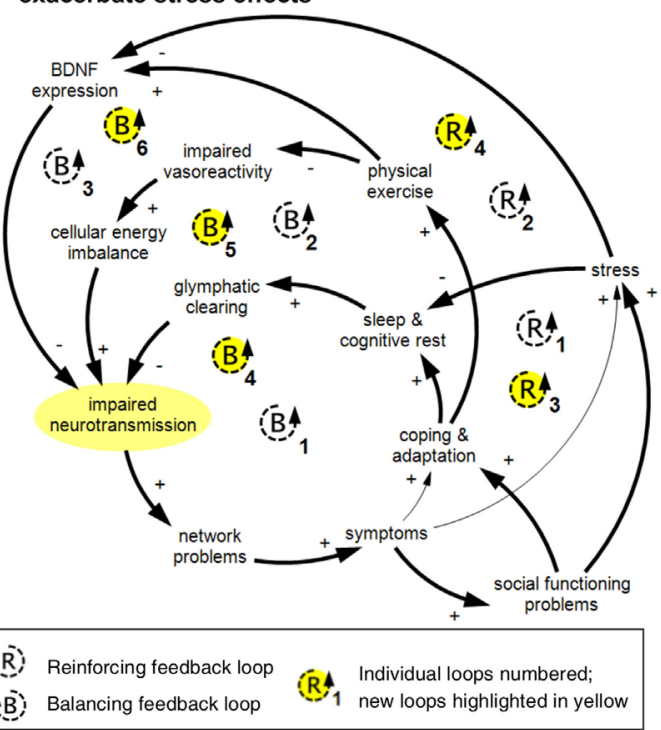

FIGURE 8 | Simplified cross-scale feedback loops pertaining to impaired neurotransmission. These diagrams depict abbreviated versions of feedback loops described in Figure 1 and demonstrate how connected loops can have compounding and counteractive effects. (A) In loop B1, impaired neurotransmission affects the function of networks; these networks and network functions include limbic, intrinsic connectivity networks, attentional filtering, and processing speed. Disruption in these networks results in a range of symptoms included in Figure $\mathbf{1}$ (specifically, light or sound sensitivity, impairment in emotional regulation, impulsivity, irritability/mood instability, stress, depression/anxiety/mood disorders, reduced social functioning, impaired working memory and executive function, reduced cognitive load capacity, dizziness and vertigo, balance and gait problems, impaired prediction of sensory input, visual/perceptual impairment, disorientation and confusion, and reduced ability to work and complete daily tasks). Either directly or indirectly, these symptoms prompt coping and adaptation strategies, including avoidance of overstimulation, pursuit of and adherence to treatment, cognitive rest, and addressing of sleep problems. Restorative sleep processes lead to glymphatic clearing of brain waste and energy byproducts, which in turn results in improved neurotransmission via an improved cellular milieu and support of neuroplasticity. (B) In loop B2, physical exercise is used as a coping and adaptation strategy, which improves vasoreactivity and cellular energy imbalance, which supports neurotransmission. In loop B3, brain-derived neutrophic factor (BDNF) expression is strengthened, which reduces impaired neurotransmission via improved neuroplasticity. (C) Stress can disrupt sleep and inhibit BDNF expression, which creates two reinforcing loops. (D) Social functioning problems can prompt coping and adaptation, which introduces three additional balancing loops, and increase stress, which compounds the reinforcing effects of stress. Diagrams rendered in MapSys.

characteristics, such as age and sex $(85,93,94)$, have complex effects that significantly shape processes at all scales, both at the time of injury and dynamically through recovery.

Injury characteristics, such as amount of force $(18,95)$, rotational vs. linear acceleration (96), and injury context (83), also affect recovery. However, each human brain is uniquely wired. Individual differences and idiosyncrasies in neural connections and networks are present from the earliest stages of neurodevelopment and differentiate further with experience (97). Even if it were possible to determine in vivo how various biomechanical 


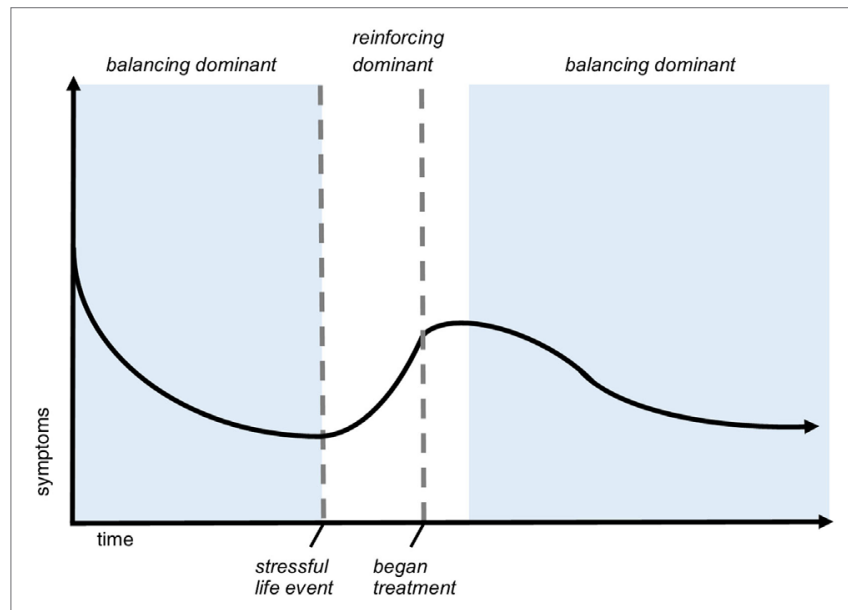

FIGURE 9 | Shifting loop dominance evidenced in trajectory of symptoms over time. This hypothetical graph of symptom severity over time demonstrates a pattern of shifting dominance of interlinked feedback loops. In the scenario, the patient is injured at $t=0$ and experiences a decreasing severity of symptoms. During this time (indicated by the first blue phase), balancing feedback is dominant. After a stressful life event, symptoms exponentially increase, meaning that reinforcing loops are dominant. Shortly after beginning treatment, balancing processes again dominate, indicated by the second blue phase. This sample recovery trajectory illustrates how feedback structure can cause nonlinear behavior of different types throughout recovery.

forces are translated from impact through the skull and layers of tissue into the human brain, it would still be difficult to make generalizations about the relationship between location of impact and function (23). At most, biomechanical studies have shown certain brain regions consistently experience some of the greatest strains and parenchymal deformations, particularly those in the subcortical white matter and its interface with cortical gray matter and the upper brainstem $(18,98)$. Outside of identifying areas generally more affected, the heterogeneity in modes of injury render most other claims of spatial localization ungeneralizable.

Many TBI studies include mixed etiologies of injuries and a wide-age range and do not control for educational and vocational backgrounds. All of these factors likely contribute unique influences at various levels in the systems models of brain injury, and none may impose a specific linear influence specifically related to outcome. Having a systems model provides a variety of options as to how to examine the multiple layers of influences at play in the evolution or resolution of symptoms following concussion.

\section{Boundaries}

System boundaries are defined in by variables with no outgoing connections, as well as elements excluded from the diagram. In the present model, the "dead end" variables are loss of consciousness, vulnerability for future injury, risk for dementia and chronic traumatic encephalopathy, reduced reaction time, nausea, and feeling out of sync. Numerous elements and relationships pertaining to concussion were excluded from the diagram, including those that did not have a clear connection to predicting long-term outcomes, provided detail that was too granular, or were redundant of existing model structure. For example, monoamines are critical to the development and maintenance of emotion-related symptoms, such as depression, anxiety, mood disorders, and stress, but given that the disruption of limbic networks appear to be the primary levers and scales of interest for concussion recovery trajectories, this molecular-level detail was excluded from the model, as it would not have provided any new connections or information about the feedback dynamics driving symptom persistence and recovery. Significant variance or detail was also collapsed into certain summary variables. For example, specific social context dynamics about return to play or school can greatly affect certain individuals, but in our model, they are summarized in the exogenous variable social pressure. Additionally, the model describes impact concussion (caused by blunt force or accelerative/decelerative forces), and excludes blast-type concussions and other injury modalities.

\section{Hubs}

In a network, hubs are nodes that are disproportionately connected with other nodes in the system $(90,99)$. The primary hub in the concussion CLD is impaired neurotransmission, which serves as a main conduit between the micro-scale left side of the diagram and the larger scales of symptoms and experience on the right side. The next most connected hubs in the diagram are stress, pathological microenvironment, and cognitive fatigue.

The concept of hubs is also important for understanding the structure of neural networks. As outlined by Bassett and Sporns (100), network neuroscience has demonstrated nonrandom topological attributes that relate to function, where neuroimaging has demonstrated "high clustering and short path length, and network communities (modules) linked by highly connected hub nodes that are in turn densely linked, forming an integrative core or rich club (p. 6)." In this context, damage to hubs can disrupt entire networks.

\section{Interventions}

A comprehensive inventory of interventions for TBI is outside the scope of this model. However, several variables for coping, adaptation, and treatment were included in the concussion CLD (Figure 1) to demonstrate the possible role of intervention processes in recovery. For example, adherence to treatment helps to reduce dyssomnias, migraine, and headache, and comorbid pain and muscle tension, as well as to increase physical exercise and cognitive rest (83). Another coping mechanism is avoidance of overstimulation, which reduces cognitive fatigue in the model. Specific treatments, such as pharmaceuticals for migraine or therapy for visual/perceptual impairment, could be customized to the individual and might be seen as exogenous drivers to the system. Future precision medicine interventions targeting subgroups of patients based on genetic profile or other aspects of physiology could introduce additional feedback loops at certain points in the model.

In a heterogeneous system, interventions should respond to individualized needs based on physical, emotional, cognitive, and social dynamics. A truism in systems science is that obvious solutions often backfire; truly effective interventions must be sensitive 
to how interrelationships between variables play out over time. For example, balancing loops can be introduced, reinforcing processes can be tempered, and drivers can be addressed. If knowledge about concussion pathophysiology becomes sophisticated enough to be able to generate a CLD specific to an individual person, customized interventions could be identified based on an analysis of system structure, including loops and drivers.

\section{DISCUSSION}

Systems models such as the one presented here provide a way for different types of knowledge from multiple subfields to be integrated into a larger working hypothesis. For concussion, the heterogeneity of the phenomenon and the diversity of methods and measures for studying it support the need for a common understanding of basic system structure. Having such a "map" of the "terrain" of knowledge about a system can be useful in both research and clinical settings.

\section{Research Applications}

As a reflection of current scientific knowledge, a systems model can facilitate the identification of research gaps and opportunities for interdisciplinary collaboration. One key observation made while building this model was the relative lack of research on restorative or ameliorative processes-particularly endogenous ones-in favor of pathological processes. Basic research into the body's diverse healing mechanisms, as well as intraindividual variability of these mechanisms, would support a more comprehensive understanding of how symptoms lessen over time and perhaps lead to new treatments (101). Analysis of the feedback structure of this model also revealed the importance of connections from the larger to smaller scales in introducing feedback loops, particularly cross-scale balancing loops that reduce symptoms. Greater understanding of cross-scale loops and other similar downscale connections would be particularly useful for improving knowledge about recovery.

A model that brings together knowledge from a diverse range of subdisciplines provides a unique opportunity to identify key components and understand the magnitude of their influence on overall clinical presentation. This framework could serve as a platform for integration of research findings regarding new diagnostic markers (e.g., blood biomarkers) or pharmaceutical treatments. Such insight about how different types of evidence contribute to a larger understanding of concussion could inform efforts to develop a new classification system for TBI. To provide the most useful input for reclassification, systems models would be developed using high-quality time-course data from a wide variety of patients as well as robust studies documenting identified pathophysiological mechanisms. Although such data are not currently available, a preliminary hypothesis model could lay the foundation for future work by encouraging such research and providing a platform for integration of different types of data.

Such a platform could be used in an iterative fashion with other research strategies such as traditional basic and clinical research and systematic reviews to work toward shared goals such as the development of clinical care guidelines. Systems approaches also serve particularly well as a complement to big-data approaches, which generate insight in a bottom-up manner directly from data, as well as personalized systems biology methods that use individual-scale "-omic" data to predict risk and recovery (102-104). When used in conjunction with these other approaches, visual systems methods such as causal-loop diagramming can serve as a valuable platform for interdisciplinary discussion, hypothesis generation and theory building, and spur methodological innovation.

Causal-loop diagrams, which depict hypothesized causal relationships between aggregate quantities, also complement current applications of network neuroscience and graph theory relating brain to neurobehavioral functioning (90). Advanced neuroimaging methods that track how networks respond to injury may ultimately prove useful in guiding treatment and outcome (105), although initial efforts have been disappointing in sports concussion (106). Systems methods might bring to this analysis useful attention to functional dynamics over time.

\section{Clinical Applications}

A systems approach can inform clinical decision-making both indirectly through the outputs of research mentioned above (i.e., clinical care guidelines, diagnostic measures, and treatment protocols) and directly by providing clinicians with a new way of viewing and analyzing concussion. As with most of medicine, clinical diagnosis, prognosis, and treatment of concussion involves pattern recognition. This model could help clinicians visualize patterns that are currently hidden in the complexity of the clinical scenario in order to better identify those individuals at high risk for poor recovery and link treatments to appropriate outcomes and measures.

Awareness of feedback mechanisms is particularly relevant in the clinic. Figure 1 illustrates how balancing processes related to sleep, cognitive rest, physical exercise, coping and adaptation, neuroplasticity, and cellular repair contribute to symptom reduction. When identifying treatment plans for concussion patients, clinicians could consider how balancing processes (e.g., sleep, cognitive rest, physical exercise, coping and adaptation, neuroplasticity, and cellular repair) might be enhanced, and how reinforcing processes (e.g., stress and social dysfunction) might be mitigated.

Another clinical takeaway is that factors endogenous to the system are more likely to persist over time. Prominent systems scientist Donella Meadows identified 12 ways to intervene in a system, organized by increasing capacity for transformative change (107). Within her framework, reorganization of system structure often has greater leverage than minor changes to variables, especially temporary or exogenously driven changes. In the clinic, this could mean helping patients to identify sustainable changes to habits, lifestyle choices, and social support that could complement and strengthen more traditional, exogenously driven therapies and treatments. For example, proper sleep hygiene and stress reduction can reduce sleep disruption, and avoidance of overstimulation and certain triggers can reduce cognitive fatigue and migraine. Endogenous means of behavioral adaptation shape the system in ongoing ways, while clinic-centered treatment can be time limited. Attention to behavioral adaptation in the clinic is 
established practice, but the causal-loop diagram provides a new rationale for this strategy.

\section{Modeling Challenges}

Modeling complex systems inevitably involves a tradeoff between comprehensiveness and legibility. The concussion model presented in Figure $\mathbf{1}$ is a qualitative representation of the modelers' understanding of the system at a certain point in time. It is, therefore, defined by the main data and themes identified during the course of research and does not include every aspect of concussion recovery.

Ideally, systems models would be living documents continually updated according to the latest medical knowledge and applied as heuristic tools in an iterative fashion. A static, two-dimensional image is also not the ideal presentation for a model with so many possible layers of information. The web-based version of the model allows users more control in navigating the model and viewing supporting information.

Model development was complicated by a lack of clarity in the literature regarding the extent to which research on TBI, broadly defined, also applies to mTBI or concussion. Whether injuries of different severities and of different types indeed belong on a single continuum is a matter of controversy (108). More precision and consistency in TBI classification, definition, and measurement would provide the basis for more clarity in findings from basic and clinical research.

The non-specificity of symptoms and the effects of litigation complicate TBI research and, therefore, model building. As outlined in recent reviews to understand vulnerabilities related to concussion outcome, consideration must include a host of both pre- as well as post-injury factors $(109,110)$. Individuals with prior neuropsychiatric conditions, especially depression, anxiety, and pain-related disorders, are particularly vulnerable to developing residual problems after a concussive brain injury. Frontal-temporal-limbic systems play a role in neuropsychiatric symptomology and are also likely injured in concussion. Neuropsychiatric symptoms also relate to symptom reporting, including symptom magnification following injury (111). Presence of litigation represents a significant issue in TBI outcome studies (112). Hiploylee and colleagues (5) demonstrate how a comprehensive approach to symptom reporting and validity testing using a longitudinal design can help control for such effects. If such methods become more commonplace, future reviews and models might preference them to identify a more precise picture of the effects of concussion.

Causal-loop diagramming does not readily depict changes in system structure over time. Both cognitive rest and physical exercise, for example, have been shown to be beneficial or harmful depending on the amount of time following injury (113). Because in-depth modeling of this subsystem was outside the scope of this project, the concussion model in Figure $\mathbf{1}$ includes only the benefits of rest and exercise. Spatial localization of injury, particularly regarding neurological damage and network disruption, is also difficult to represent with a diagram methodology structured around aggregate quantities.

Many of the challenges faced in modeling concussion-such as classification uncertainty, heterogeneity, and diversity of measures-reflect challenges stemming from the complexity of concussion itself that are present, but not always fully acknowledged, in traditional TBI research. For example, the failure of promising Phase III clinical trials for TBI treatments may say more about the lack of precision in the definition and measurement of TBI than the potential effectiveness of the proposed treatments for particular subgroups (114).

\section{Future Work}

The model described in this article is a demonstration of an innovative methodology and serves as a proof of concept for future systems-oriented efforts to understand TBI. This model could be further developed to include a more detailed depiction of certain subsystems, particularly at the network, experiential, and social scales. As scientific knowledge about concussion expands, the model could be adapted to reflect the changing consensus. Such a "living model" would more accurately depict the current state of knowledge about concussion and would, therefore, provide a more timely basis for clinical and research applications. The web-based version of Figure 1, which enables public commenting on specific model elements and relationships, is a step in this direction. However, procedures and resources for updating such a model on an ongoing basis have not yet been determined.

Future modeling efforts could include the development of computational system dynamics models based on the concussion CLD. System dynamics models are a logical extension of CLDs and introduce the dimension of time to the model, which allows for a more sophisticated examination of recovery trajectories and leverage points based on the operationalization of variables. Feedback mechanisms and the influence of personal and injury characteristics could, therefore, be analyzed in more detail. Specification and testing of these models, however, requires timecourse data of key system variables, which is currently lacking in TBI research.

Specific hypotheses identified using this model could also be tested using other methods. For example, research to test the hypothesis that downscale connections have outsize influence on system behavior could be conducted either experimentally or using a computational model. Neuroimaging-based neural networks could also be derived that correspond to some of the hypothetical networks depicted in Figure 1.

\section{CONCLUSION}

This research has shown that applying systems methods to concussion yields insight that is applicable in both research and clinical settings. Identifying key loops and drivers and considering how loop dominance may shift, either from endogenous or exogenous factors, is crucial to understanding the ways that post-concussive symptoms persist or resolve over time. By using systems modeling in conjunction with other new and more traditional approaches, a potentially fruitful new area of research could provide the synthesis and analysis necessary to address the heterogeneity and complexity found in concussion-a crucial step toward improving clinical outcomes. 


\section{AUTHOR CONTRIBUTIONS}

EK was the lead modeler and lead author. EP and WW contributed substantially to the model and article. EK, EP, and WW conducted expert interviews and literature review. EB, DW, $\mathrm{ML}, \mathrm{JC}, \mathrm{GH}$, and WG provided critical review and revision of the model and article. WW also provided systems modeling guidance.

\section{ACKNOWLEDGMENTS}

The authors would like to thank the Brain Trauma Foundation, the Brain Trauma Evidence-based Consortium, and the associated team of expert clinicians and researchers for iterative feedback and support during this multi-year collaboration. The authors would also like to thank the TBI experts they interviewed, as well as Nancy Carney for initiating this project.

\section{FUNDING}

This material is based upon work supported by the US Army Contracting Command, Aberdeen Proving Ground, Natick Contracting Division, through a contract awarded to Stanford

\section{REFERENCES}

1. McCrory P, Meeuwisse W, Dvořák J, Aubry M, Bailes J, Broglio S, et al. Consensus statement on concussion in sport-the 5th International conference on concussion in sport held in Berlin. Br J Sports Med (2017) 51(11): 838-47. doi:10.1136/bjsports-2017-097878

2. Raskin SA, Lovejoy DW, Stevens MC, Zamroziewicz M, Oakes HJ. Mild traumatic brain injury. In: Levin HS, Shum DHK, Chan RCK, editors. Understanding Traumatic Brain Injury: Current Research and Future Directions. New York: Oxford University Press (2014). p. 370-412.

3. Katz DI, Cohen SI, Alexander MP. Mild traumatic brain injury. Handb Clin Neurol (2015) 127:131-56. doi:10.1016/B978-0-444-52892-6.00009-X

4. Hawryluk GWJ, Bullock MR. Design of acute neuroprotection studies. Handb Clin Neurol (2015) 128:761-78. doi:10.1016/B978-0-444-63521-1.00047-9

5. Hiploylee C, Dufort PA, Davis HS, Wennberg RA, Tartaglia MC, Mikulis D, et al. longitudinal study of postconcussion syndrome: not everyone recovers. J Neurotrauma (2017) 34(8):1511-23. doi:10.1089/neu.2016.4677

6. Lingsma HF, Roozenbeek B, Steyerberg EW, Murray GD, Maas AI. Early prognosis in traumatic brain injury: from prophecies to predictions. Lancet Neurol (2010) 9(5):543-54. doi:10.1016/S1474-4422(10)70065-X

7. Maas AIR, Lingsma HF, Roozenbeek B. Predicting outcome after traumatic brain injury. Handb Clin Neurol (2015) 128:455-74. doi:10.1016/B9780-444-63521-1.00029-7

8. Hack D. TBI clinical trials: past, present, and future. Bridge Natl Acad Eng (2016) 46(1):34-8

9. Margulies S, Hicks R. Combination Therapies for Traumatic Brain Injury: Prospective Considerations. J Neurotrauma (2009) 26:925-39. doi:10.1089/ neu.2008-0794

10. Mayer AR, Quinn DK, Master CL. The spectrum of mild traumatic brain injury: a review. Neurology (2017) 89:1-10. doi:10.1212/WNL.0000000000004214

11. Stein DG, Geddes RI, Sribnick EA. Recent developments in clinical trials for the treatment of traumatic brain injury. Handb Clin Neurol (2015) 127: 433-51. doi:10.1016/B978-0-444-52892-6.00028-3

12. Hawryluk GWJ, Manley GT. Classification of traumatic brain injury: past, present, and future. Handb Clin Neurol (2015) 127:15-21. doi:10.1016/ B978-0-444-52892-6.00002-7

13. Carroll LJ, Cassidy JD, Holm L, Kraus J, Coronado VG; WHO Collaborating Centre Task Force on Mild Traumatic Brain Injury. Methodological issues
University (W911QY-14-C-0086), and a subcontract awarded to the Brain Trauma Foundation. This material is in part the result of work supported with resources and the use of facilities at the VA Portland Health Care System, the VA Career Development Award \#IK2 BX002712, and Portland VA Research Foundation (MML). In part, the Brain Imaging and Behavior Lab that EB directs was supported by the Army Medical Research and Material Command under Award No. W81XWH-13-2-0095 (Chronic Effects of Neurotrauma Consortium). Also, EB co-directs the Neuropsychological Assessment and Research Laboratory at Brigham Young University which includes forensic consultation. Any opinions, findings, and conclusions or recommendations expressed in this material are those of the author(s) and do not necessarily reflect the views of the US Army Contracting Command, Aberdeen Proving Ground, Natick Contracting Division, Stanford University, the Brain Trauma Foundation, U.S. Department of Veterans Affairs or the United States Government.

\section{SUPPLEMENTARY MATERIAL}

The Supplementary Material for this article can be found online at https://www.frontiersin.org/articles/10.3389/fneur.2018.00203/ full\#supplementary-material.

and research recommendations for mild traumatic brain injury: the WHO collaborating centre task force on mild traumatic brain injury. J Rehabil Med (2004) (43 Suppl):113-25. doi:10.1080/16501960410023877

14. Cassidy JD. Epidemiology of mild brain injury: quality of the scientific evidence. J Neurol Neurosurg Psychiatry (2010) 81(10):e12-12. doi:10.1136/ jnnp.2010.217554.28

15. Wright DW, Yeatts SD, Silbergleit R, Palesch YY, Hertzberg VS, Frankel M, et al. Very Early Administration of Progesterone for Acute Traumatic Brain Injury. N Engl J Med (2014) 371:2457-66. doi:10.1056/NEJMoa1404304

16. McGinn MJ, Povlishock JT. Cellular and molecular mechanisms of injury and spontaneous recovery. Handb Clin Neurol (2015) 127:67-87. doi:10.1016/ B978-0-444-52892-6.00005-2

17. Blennow K, Hardy J, Zetterberg H. The neuropathology and neurobiology of traumatic brain injury. Neuron (2012) 76(5):886-99. doi:10.1016/j.neuron. 2012.11.021

18. Hemphill MA, Dauth S, Yu CJ, Dabiri BE, Parker KK. Traumatic brain injury and the neuronal microenvironment: a potential role for neuropathological mechanotransduction. Neuron (2015) 85(6):1177-92. doi:10.1016/j.neuron. 2015.02.041

19. Kenzie ES, Parks EL, Bigler ED, Lim MM, Chesnutt JC, Wakeland W. Concussion as a multi-scale complex system: an interdisciplinary synthesis of current knowledge. Front Neurol (2017) 8:513. doi:10.3389/fneur.2017.00513

20. Marklund N, Hillered L. Animal modelling of traumatic brain injury in preclinical drug development: where do we go from here? Br J Pharmacol (2011) 164(4):1207-29. doi:10.1111/j.1476-5381.2010.01163.x

21. Rosenbaum SB, Lipton ML. Embracing chaos: the scope and importance of clinical and pathological heterogeneity in mTBI. Brain Imaging Behav (2012) 6(2):255-82. doi:10.1007/s11682-012-9162-7

22. Maas AIR, Menon DK, Adelson PD, Andelic N, Bell MJ, Belli A, et al. Traumatic brain injury: integrated approaches to improve prevention, clinical care, and research. Lancet Neurol (2017) 16(12):987-1048. doi:10.1016/ S1474-4422(17)30371-X

23. McAllister TW, Arciniegas D. Evaluation and treatment of postconcussive symptoms. NeuroRehabilitation (2002) 17(4):265-84.

24. Stone JR, Wilde EA, Taylor BA, Tate DF, Levin H, Bigler ED, et al. Supervised learning technique for the automated identification of white matter hyperintensities in traumatic brain injury. Brain Inj (2016) 30(12):1458-68. doi:10.1080/02699052.2016.1222080 
25. Silverberg ND, Gardner AJ, Brubacher JR, Panenka WJ, Li JJ, Iverson GL. Systematic review of multivariable prognostic models for mild traumatic brain injury. J Neurotrauma (2014) 32(8):517-26. doi:10.1089/neu. 2014.3600

26. Wäljas M, Lange RT, Hakulinen U, Huhtala H, Dastidar P, Hartikainen K, et al. Biopsychosocial outcome after uncomplicated mild traumatic brain injury. J Neurotrauma (2013) 31(1):108-24. doi:10.1089/neu.2013.2941

27. Wäljas M, Iverson GL, Lange RT, Hakulinen U, Dastidar P, Huhtala H, et al. A prospective biopsychosocial study of the persistent post-concussion symptoms following mild traumatic brain injury. J Neurotrauma (2014) 32(8):534-47. doi:10.1089/neu.2014.3339

28. Mabry PL, Kaplan RM. Systems science: a good investment for the public's health. Health Educ Behav (2013) 40(1 Suppl):9S-12S. doi:10.1177/ 1090198113503469

29. Sterman JD. Learning from evidence in a complex world. Am J Public Health (2006) 96(3):505-14. doi:10.2105/AJPH.2005.066043

30. Mabry PL, Olster DH, Morgan GD, Abrams DB. Interdisciplinarity and systems science to improve population health. Am J Prev Med (2008) 35(2): S211-24. doi:10.1016/j.amepre.2008.05.018

31. Johnston LM, Matteson CL, Finegood DT. Systems science and obesity policy: a novel framework for analyzing and rethinking population-level planning. Am J Public Health (2014) 104(7):1270-8. doi:10.2105/AJPH. 2014.301884

32. Wakeland W, Nielsen A, Geissert P. Dynamic model of nonmedical opioid use trajectories and potential policy interventions. Am J Drug Alcohol Abuse (2015) 41(6):508-18. doi:10.3109/00952990.2015.1043435

33. Wittenborn AK, Rahmandad H, Rick J, Hosseinichimeh N. Depression as a systemic syndrome: mapping the feedback loops of major depressive disorder. Psychol Med (2016) 46(03):551-62. doi:10.1017/S0033291715002044

34. Carney N, Ghajar J, Jagoda A, Bedrick S, Davis-O'Reilly C, duCoudray H, et al. Concussion guidelines step 1: systematic review of prevalent indicators. Neurosurgery (2014) 75:S3-15. doi:10.1227/NEU.0000000000000433

35. Sterman JD. System dynamics modeling: tools for learning in a complex world. Calif Manage Rev (2001) 43(4):8-25. doi:10.2307/41166098

36. Jones N, Ross H, Lynam T, Perez P, Leitch A. Mental models: an interdisciplinary synthesis of theory and methods. Ecol Soc (2011) 16(1). doi:10.5751/ ES-03802-160146

37. Bandak FA, Ling G, Bandak A, De Lanerolle NC. Injury biomechanics, neuropathology, and simplified physics of explosive blast and impact mild traumatic brain injury. Handb Clin Neurol (2015) 127:89-104. doi:10.1016/ B978-0-444-52892-6.00006-4

38. Chen Y, Huang W, Constantini S. The differences between blast-induced and sports-related brain injuries. Front Neurol (2013) 4. doi:10.3389/fneur. 2013.00119

39. Ling G, Ecklund JM, Bandak FA. Brain injury from explosive blast: description and clinical management. Handb Clin Neurol (2015) 127:173-80. doi:10.1016/B978-0-444-52892-6.00011-8

40. Kumu Inc. (2018). Available from: https://kumu.io/ (Accessed: March 24, 2018).

41. Smith DH, Hicks R, Povlishock JT. Therapy development for diffuse axonal injury. J Neurotrauma (2013) 30(5):307-23. doi:10.1089/neu.2012.2825

42. Giza CC, Hovda DA. The new neurometabolic cascade of concussion. Neurosurgery (2014) 75:S24-33. doi:10.1227/NEU.0000000000000505

43. Browne KD, Chen X-H, Meaney DF, Smith DH. Mild traumatic brain injury and diffuse axonal injury in swine. J Neurotrauma (2011) 28(9):1747-55. doi:10.1089/neu.2011.1913

44. Johnson VE, Stewart W, Smith DH. Axonal pathology in traumatic brain injury. Exp Neurol (2013) 246:35-43. doi:10.1016/j.expneurol.2012.01.013

45. Calabrese F, Rossetti AC, Racagni G, Gass P, Riva MA, Molteni R. Brainderived neurotrophic factor: a bridge between inflammation and neuroplasticity. Front Cell Neurosci (2014) 8:430. doi:10.3389/fncel.2014.00430

46. Mayer CL, Huber BR, Peskind E. Traumatic brain injury, neuroinflammation, and post-traumatic headaches. Headache (2013) 53(9):1523-30. doi:10.1111/ head. 12173

47. Lafrenaye AD, Todani M, Walker SA, Povlishock JT. Microglia processes associate with diffusely injured axons following mild traumatic brain injury in the micro pig. J Neuroinflammation (2015) 12:186. doi:10.1186/ s12974-015-0405-6
48. Asken BM, DeKosky ST, Clugston JR, Jaffee MS, Bauer RM. Diffusion tensor imaging (DTI) findings in adult civilian, military, and sport-related mild traumatic brain injury (mTBI): a systematic critical review. Brain Imaging Behav (2017):1-28. doi:10.1007/s11682-017-9708-9

49. Fisher JAN, Huang S, Ye M, Nabili M, Wilent WB, Krauthamer V, et al. Real-time detection and monitoring of acute brain injury utilizing evoked electroencephalographic potentials. IEEE Trans Neural Syst Rehabil Eng (2016) 24(9):1003-12. doi:10.1109/TNSRE.2016.2529663

50. Giza CC, Hovda DA. The neurometabolic cascade of concussion. JAthl Train (2001) 36(3):228-35.

51. Oehr L, Anderson J. Diffusion-tensor imaging findings and cognitive function following hospitalised mixed-mechanism mild traumatic brain injury: a systematic review and meta-analysis. Arch Phys Med Rehabil (2017) 98(11):2308-19. doi:10.1016/j.apmr.2017.03.019

52. Smith DH. Neuromechanics and pathophysiology of diffuse axonal injury in concussion. Bridge Natl Acad Eng (2016) 46(1):79-84.

53. Hwang K, Bertolero MA, Liu WB, D'Esposito M. The human thalamus is an integrative hub for functional brain networks. J Neurosci (2017) 37(23): 5594-607. doi:10.1523/JNEUROSCI.0067-17.2017

54. Cohen JR, D'Esposito M. The segregation and integration of distinct brain networks and their relationship to cognition. J Neurosci (2016) 36(48): 12083-94. doi:10.1523/JNEUROSCI.2965-15.2016

55. Dulla CG, Coulter DA, Ziburkus J. From molecular circuit dysfunction to disease: case studies in epilepsy, traumatic brain injury, and Alzheimer's disease. Neuroscientist (2016) 22(3):295-312. doi:10.1177/1073858415585108

56. Simon DW, McGeachy MJ, Bayır H, Clark RSB, Loane DJ, Kochanek PM. The far-reaching scope of neuroinflammation after traumatic brain injury. Nat Rev Neurol (2017) 13(3):171-91. doi:10.1038/nrneurol.2017.13

57. Zetterberg H, Smith DH, Blennow K. Biomarkers of mild traumatic brain injury in cerebrospinal fluid and blood. Nat Rev Neurol (2013) 9(4):201-10. doi:10.1038/nrneurol.2013.9

58. Stamm JM, Bourlas AP, Baugh CM, Fritts NG, Daneshvar DH, Martin BM, et al. Age of first exposure to football and later-life cognitive impairment in former NFL players. Neurology (2015) 84(11):1114-20. doi:10.1212/WNL. 0000000000001358

59. Sours C, Zhuo J, Janowich J, Aarabi B, Shanmuganathan K, Gullapalli RP. Default mode network interference in mild traumatic brain injury - a pilot resting state study. Brain Res (2013) 1537:201-15. doi:10.1016/j.brainres. 2013.08.034

60. Logsdon AF, Lucke-Wold BP, Turner RC, Huber JD, Rosen CL, Simpkins JW. Role of microvascular disruption in brain damage from traumatic brain injury. Compr Physiol (2015) 5(3):1147-60. doi:10.1002/cphy.c140057

61. Hillary FG, Grafman JH. Injured brains and adaptive networks: the benefits and costs of hyperconnectivity. Trends Cogn Sci (2017) 21(5):385-401. doi:10.1016/j.tics.2017.03.003

62. Heine L, Soddu A, Gómez F, Vanhaudenhuyse A, Tshibanda L, Thonnard M, et al. Resting state networks and consciousness: alterations of multiple resting state network connectivity in physiological, pharmacological, and pathological consciousness states. Front Psychol (2012) 3:295. doi:10.3389/ fpsyg.2012.00295

63. Jiang H, White MP, Greicius MD, Waelde LC, Spiegel D. Brain activity and functional connectivity associated with hypnosis. Cereb Cortex (2017) 27(8):4083-93. doi:10.1093/cercor/bhw220

64. Raichle ME, MacLeod AM, Snyder AZ, Powers WJ, Gusnard DA, Shulman GL. A default mode of brain function. Proc Natl Acad Sci U S A (2001) 98(2): 676-82. doi:10.1073/pnas.98.2.676

65. Bigler ED. Default mode network, connectivity, traumatic brain injury and post-traumatic amnesia. Brain J Neurol (2016) 139(Pt 12):3054-7. doi:10.1093/brain/aww277

66. Barbey AK, Belli A, Logan A, Rubin R, Zamroziewicz M, Operskalski JT. Network topology and dynamics in traumatic brain injury. Curr Opin Behav Sci (2015) 4:92-102. doi:10.1016/j.cobeha.2015.04.002

67. Han K, Mac Donald CL, Johnson AM, Barnes Y, Wierzechowski L, Zonies D, et al. Disrupted modular organization of resting-state cortical functional connectivity in U.S. military personnel following concussive "mild" blastrelated traumatic brain injury. Neuroimage (2014) 84:76-96. doi:10.1016/j. neuroimage.2013.08.017 
68. Johnson VE, Stewart W, Smith DH. Widespread tau and amyloid-beta pathology many years after a single traumatic brain injury in humans: longterm AD-like pathology after single TBI. Brain Pathol (2012) 22(2):142-9. doi:10.1111/j.1750-3639.2011.00513.x

69. Shumskaya E, Andriessen TMJC, Norris DG, Vos PE. Abnormal whole-brain functional networks in homogeneous acute mild traumatic brain injury. Neurology (2012) 79(2):175-82. doi:10.1212/WNL.0b013e31825f04fb

70. van den Heuvel MP, Sporns O. Rich-club organization of the human connectome. J Neurosci (2011) 31(44):15775-86. doi:10.1523/JNEUROSCI.353911.2011

71. Ghajar J, Ivry RB; Cognitive and Neurobiological Research Consortium. The predictive brain state: timing deficiencyin traumaticbrain injury? Neurorehabil Neural Repair (2008) 22(3):217-27. doi:10.1177/1545968308315600

72. Hladky SB, Barrand MA. Metabolite Clearance During Wakefulness and Sleep. In: Barrett JE, editor. Handbook of Experimental Pharmacology. Berlin; Heidelberg: Springer (2017). doi:10.1007/164_2017_37

73. Iliff JJ, Chen MJ, Plog BA, Zeppenfeld DM, Soltero M, Yang L, et al. Impairment of glymphatic pathway function promotes tau pathology after traumatic brain injury. J Neurosci (2014) 34(49):16180-93. doi:10.1523/ JNEUROSCI.3020-14.2014

74. Xie L, Kang H, Xu Q, Chen MJ, Liao Y, Thiyagarajan M, et al. Sleep drives metabolite clearance from the adult brain. Science (2013) 342(6156):373-7. doi:10.1126/science.1241224

75. Malhotra RK. Sleep, recovery, and performance in sports. Neurol Clin (2017) 35(3):547-57. doi:10.1016/j.ncl.2017.03.002

76. Davidson RJ. Well-being and affective style: neural substrates and biobehavioural correlates. Philos Trans R Soc B Biol Sci (2004) 359(1449):1395-411. doi:10.1098/rstb.2004.1510

77. Fonzo GA, Etkin A. Affective neuroimaging in generalized anxiety disorder: an integrated review. Dialogues Clin Neurosci (2017) 19(2):169-79.

78. Yamawaki S, Okada G, Okamoto Y, Liberzon I. Mood dysregulation and stabilization: perspectives from emotional cognitive neuroscience. Int $J$ Neuropsychopharmacol (2012) 15(5):681-94. doi:10.1017/S1461145711000757

79. Sacher J, Neumann J, Fünfstück T, Soliman A, Villringer A, Schroeter ML. Mapping the depressed brain: a meta-analysis of structural and functional alterations in major depressive disorder. J Affect Disord (2012) 140(2):142-8. doi:10.1016/j.jad.2011.08.001

80. Lee SW, Gerdes L, Tegeler CL, Shaltout HA, Tegeler CH. A bihemispheric autonomic model for traumatic stress effects on health and behavior. Front Psychol (2014) 5. doi:10.3389/fpsyg.2014.00843

81. Gordon WA, Cantor J, Kristen D, Tsaousides T. Long-term social integration and community support. Handb Clin Neurol (2015) 127:423-31. doi:10.1016/ B978-0-444-52892-6.00027-1

82. Howell DR, Osternig LR, Koester MC, Chou L-S. The effect of cognitive task complexity on gait stability in adolescents following concussion. Exp Brain Res (2014) 232(6):1773-82. doi:10.1007/s00221-014-3869-1

83. Reynolds E, Collins MW, Mucha A, Troutman-Ensecki C. Establishing a clinical service for the management of sports-related concussions. Neurosurgery (2014) 75:S71-81. doi:10.1227/NEU.0000000000000471

84. Astafiev SV, Zinn KL, Shulman GL, Corbetta M. Exploring the physiological correlates of chronic mild traumatic brain injury symptoms. Neuroimage Clin (2016) 11:10-9. doi:10.1016/j.nicl.2016.01.004

85. King LA, Horak FB, Mancini M, Pierce D, Priest KC, Chesnutt J, et al. Instrumenting the balance error scoring system for use with patients reporting persistent balance problems after mild traumatic brain injury. Arch Phys Med Rehabil (2014) 95(2):353-9. doi:10.1016/j.apmr.2013.10.015

86. Sullivan KA, Kempe CB, Edmed SL, Bonanno GA. Resilience and other possible outcomes after mild traumatic brain injury: a systematic review. Neuropsychol Rev (2016) 26(2):173-85. doi:10.1007/s11065-016-9317-1

87. Losoi H, Silverberg ND, Wäljas M, Turunen S, Rosti-Otajärvi E, Helminen $\mathrm{M}$, et al. Resilience is associated with outcome from mild traumatic brain injury. J Neurotrauma (2015) 32(13):942-9. doi:10.1089/neu.2014.3799

88. Rathbone A, Tharmaradinam S, Jiang S, Rathbone M, Kumbhare DA. A review of the neuro- and systemic inflammatory responses in post concussion symptoms: introduction of the "post-inflammatory brain syndrome" PIBS. Brain Behav Immun (2015) 46:1-16. doi:10.1016/j.bbi.2015.02.009

89. Su Y, Fan W, Ma Z, Wen X, Wang W, Wu Q, et al. Taurine improves functional and histological outcomes and reduces inflammation in traumatic brain injury. Neuroscience (2014) 266:56-65. doi:10.1016/j.neuroscience.2014.02.006
90. Sporns O, Betzel RF. Modular brain networks. Annu Rev Psychol (2016) 67(1):613-40. doi:10.1146/annurev-psych-122414-033634

91. Bigler ED, Stern Y. Traumatic brain injury and reserve. In: Grafman J, Salazar AM, editors. Handbook of Clinical Neurology (Vol. 128, Part II), Amsterdam: Elsevier (2015). p. 691-710.

92. McCarty CA, Zatzick D, Stein E, Wang J, Hilt R, Rivara FP, et al. Collaborative care for adolescents with persistent postconcussive symptoms: a randomized trial. Pediatrics (2016) 138(4):e20160459. doi:10.1542/peds.2016-0459

93. Dougan BK, Horswill MS, Geffen GM. Athletes' age, sex, and years of education moderate the acute neuropsychological impact of sports-related concussion: a meta-analysis. J Int Neuropsychol Soc (2014) 20(01):64-80. doi:10.1017/S1355617712001464

94. Crowe LM, Catroppa C, Andersen V. Sequelae in children: developmental consequences. In: Salazar AM, editor. Handbook of Clinical Neurology: Traumatic Brain Injury Part II. 3rd ed. (Vol. 128), Elsevier (2015). p. 661-77.

95. Cloots RJH, van Dommelen JAW, Kleiven S, Geers MGD. Multi-scale mechanics of traumatic brain injury: predicting axonal strains from head loads. Biomech Model Mechanobiol (2013) 12(1):137-50. doi:10.1007/s10237012-0387-6

96. Meaney DF, Smith DH. Biomechanics of concussion. Clin Sports Med (2011) 30(1):19-31. doi:10.1016/j.csm.2010.08.009

97. Seung S. Connectome: How the Brain's Wiring Makes Us Who We Are. Boston: Mariner Books (2013). 384 p.

98. Ganpule S, Daphalapurkar NP, Ramesh KT, Knutsen AK, Pham DL, Bayly $\mathrm{PV}$, et al. A three-dimensional computational human head model that captures live human brain dynamics. J Neurotrauma (2017) 34(13):2154-66. doi:10.1089/neu.2016.4744

99. Crossley NA, Fox PT, Bullmore ET. Meta-connectomics: human brain network and connectivity meta-analyses. Psychol Med (2016) 46(5):897-907. doi:10.1017/S0033291715002895

100. Bassett DS, Sporns O. Network neuroscience. Nat Neurosci (2017) 20(3): 353-64. doi:10.1038/nn.4502

101. Muresanu DF, Buzoianu A, Florian SI, von Wild T. Towards a roadmap in brain protection and recovery. J Cell Mol Med (2012) 16(12):2861-71. doi:10.1111/j.1582-4934.2012.01605.x

102. Insel TR, Cuthbert BN. Brain disorders? Precisely. Science (2015) 348(6234):499-500. doi:10.1126/science.aab2358

103. Dougherty JD, Yang C, Lake AM. Systems biology in the central nervous system: a brief perspective on essential recent advancements. Curr Opin Syst Biol (2017) 3:67-76. doi:10.1016/j.coisb.2017.04.011

104. Kurowski BG, Treble-Barna A, Pitzer AJ, Wade SL, Martin LJ, Chima RS, et al. Applying systems biology methodology to identify genetic factors possibly associated with recovery after traumatic brain injury. J Neurotrauma (2017) 34(14):2280-90. doi:10.1089/neu.2016.4856

105. Yuan W, Wade SL, Quatman-Yates C, Hugentobler JA, Gubanich PJ, Kurowski BG. Structural connectivity related to persistent symptoms after mild TBI in adolescents and response to aerobic training: preliminary investigation. J Head Trauma Rehabil (2017) 32(6):378-84. doi:10.1097/ HTR.0000000000000318

106. Broglio SP, McCrea M, McAllister T, Harezlak J, Katz B, Hack D, et al. A National study on the effects of concussion in collegiate athletes and US military service academy members: the NCAA-DoD concussion assessment, research and education (CARE) consortium structure and methods. Sports Med (2017) 47(7):1437-51. doi:10.1007/s40279-017-0707-1

107. Meadows DH. Thinking in Systems: A Primer. White River Junction, VT: Chelsea Green Publishing (2007). 182 p.

108. Slobounov S, Gay M, Johnson B, Zhang K. Concussion in athletics: ongoing clinical and brain imaging research controversies. Brain Imaging Behav (2012) 6(2):224-43. doi:10.1007/s11682-012-9167-2

109. Silver JM, McAllister TW, Arciniegas DB. Depression and cognitive complaints following mild traumatic brain injury. Am J Psychiatry (2009) 166(6):653-61. doi:10.1176/appi.ajp.2009.08111676

110. Arciniegas DB, Anderson CA, Topkoff J, McAllister TW. Mild traumatic brain injury: a neuropsychiatric approach to diagnosis, evaluation, and treatment. Neuropsychiatr Dis Treat (2005) 1(4):311-27.

111. Jackson CE, Nordstrom L, Fonda JR, Fortier CB, Milberg WP, McGlinchey RE. Reporting of symptoms associated with concussion by OEF/OIF/OND Veterans: comparison between research and clinical contexts. Brain Inj (2017) 31(4):485-92. doi:10.1080/02699052.2017.1280740 
112. Lippa SM, Lange RT, French LM, Iverson GL. Performance validity, neurocognitive disorder, and post-concussion symptom reporting in service members with a history of mild traumatic brain injury. Arch Clin Neuropsychol (2017) 1:1-13. doi:10.1093/arclin/acx098

113. Leddy JJ, Kozlowski K, Fung M, Pendergast DR, Willer B. Regulatory and autoregulatory physiological dysfunction as a primary characteristic of post concussion syndrome: implications for treatment. NeuroRehabilitation (2007) 22(3):199-205.

114. Stein DG. Embracing failure: what the phase III progesterone studies can teach about TBI clinical trials. Brain Inj (2015) 29(11):1259-72. doi:10.3109/ 02699052.2015 .1065344
Conflict of Interest Statement: The authors declare that the research was conducted in the absence of any commercial or financial relationships that could be construed as a potential conflict of interest.

Copyright (C) 2018 Kenzie, Parks, Bigler, Wright, Lim, Chesnutt, Hawryluk, Gordon and Wakeland. This is an open-access article distributed under the terms of the Creative Commons Attribution License (CC BY). The use, distribution or reproduction in other forums is permitted, provided the original author(s) and the copyright owner are credited and that the original publication in this journal is cited, in accordance with accepted academic practice. No use, distribution or reproduction is permitted which does not comply with these terms. 